\title{
Design of Network and Node for Discrete Manufacturing Information Monitoring
}

\author{
Chen Dongfang \\ ${ }^{1}$ School of Mechatronical Engineering Beijing Institute \\ of Technology \\ ${ }^{2}$ Beijing Tiandi-Marco Electro-Hydraulic Contol \\ Systems Co., Ltd. \\ Beijing, China. \\ chendongfang79@163.com \\ Pu Mingsong \\ Beijing Tiandi-Marco Electro-Hydraulic Contol \\ Systems Co., Ltd. \\ Beijing, China. \\ pums@tdmarco.com
}

\author{
Wang Tianying \\ Beijing Tiandi-Marco Electro-Hydraulic Contol \\ Systems Co., Ltd. \\ Beijing , China. \\ wty@tdmarco.com
}

\begin{abstract}
This paper presents a design for information gathering networks and nodes for discrete manufacturing information monitoring. Firstly, the wireless network characteristics and categories are analyzed. The paper then proposes a combined wireless network with high speed WIFI and low speed ZigBee network to collect information. Requirements for production information monitoring are then analyzed. A monitoring node is designed based on the ZigBee protocol network. The software of the monitoring node and the hardware are designed based on functional requirements analysis. Then the discrete manufacturing network node is designed. Finally, An experiment was performed to test the actual network by discrete manufacturing way. Results show that the network and node performed effectively. Requirements of the actual network to collect information were fully satisfied. The nodes are more convenient, and easy maintenance, and low cost. the mix network strategies will play a more important role in the future in discrete manufacturing industry.
\end{abstract}

Keywords-component; ZigBee protocol; Discrete manufacturing; Information monitoring; Wireless transmission; MES system

\section{INTRODUCTION}

Recently, Manufacturing Execution Systems (MES) are being used for a growing number of applications in the manufacturing industry. Due to the site conditions of a manufacturing environment, data acquisition methods underlying data collection have always been a difficult problem. In order to solve the problem of data acquisition, this paper focuses on the information monitoring network and node analysis and design, to improve the underlying data collection.

In an actual production environment, most of the enterprise production methods are discrete manufacturing jobs with various product and process types, and jobs may be even scattered within the same factory site. The quality of the production process information is difficult to record.
For real time data collection and monitoring of production quality, control of the production schedule causes big difficulties. The research in [1-3] describes the discrete data collection problems in production processes. The main technology used is RFID technology, namely active or passive tags on each product which contain tag identity information, and enable the production status information to be identified. Compared with bar code information, the disadvantage of RFID technology is the high label cost. Additionally, RFID is not very suitable for larger volumes of information collection and transmission, such as picture or video information collection and transmission, etc.

Due to the characteristic that discrete information is scattered throughout the production process, a wireless network method is suitable for data acquisition for information monitoring. There are numerous wireless technologies currently used for this application: wireless WIFI network, RFID network, wireless ZigBee protocol network, bluetooth, infrared, etc. However, wireless WIFI and the ZigBee protocol network are the most popular applications

Wireless WIFI network technology is now capable of short distance communication and is frequently used for large data transmission traffic. There is some related research on applying wireless WIFI networks to production process information acquisition. A study of this type is given in [4], which is based on the technology of wireless access terminals, which are used to analyze the production data and the information collected. Wireless WIFI technology for practical applications has an obvious communication transmission speed advantage, but power consumption is larger than ZigBee, and it has limited flexibility as a mobile terminal in network applications. An alternative is to use the ZigBee protocol for wireless sensor networks (WSN), which can realize large-scale networks with automatic network and node functionality. ZigBee is developed based on IEEE 802.15.4 [5] and its characteristics are: 1) low power consumption and 2) 
nodes have the ability to be expanded. The ZigBee protocol in the $2.4 \mathrm{GHz}$ band is suitable for small-scale data communication; the data transmission rate is low and the distribution range is small with a low cost association [6-9].

Based on the above analysis, this paper analyzes how to obtain monitoring information in the production process. Two types of network are proposed for the production process information acquisition. For high-speed traffic information collection, the WIFI network can be used. For low speed disperse information collection, the ZigBee protocol network is suitable. A network based on ZigBee protocol monitor node hardware is designed. Finally, the ZigBee network is tested for the process of manufacturing products.

\section{NETWORK MONITORING AND CONTROL SYSTEM TYPE STYLE AND FONTS}

\section{A. Network monitoring system and functional description}

In an MES system, there are various pieces of production information that need to be collected, to achieve an accurate overview of the MES system and maintain real-time performance. As discussed previously, two wireless networks are designed to be used in information monitoring systems in discrete manufacturing processes: the wireless WIFI network (Fig .1) and the ZigBee wireless network (Fig .2).

As shown in Fig .1, a wireless WIFI network can perform batch production process information data collection at a high speed. The wireless network includes the terminal, wireless AP, wireless routing and the Ethernet.

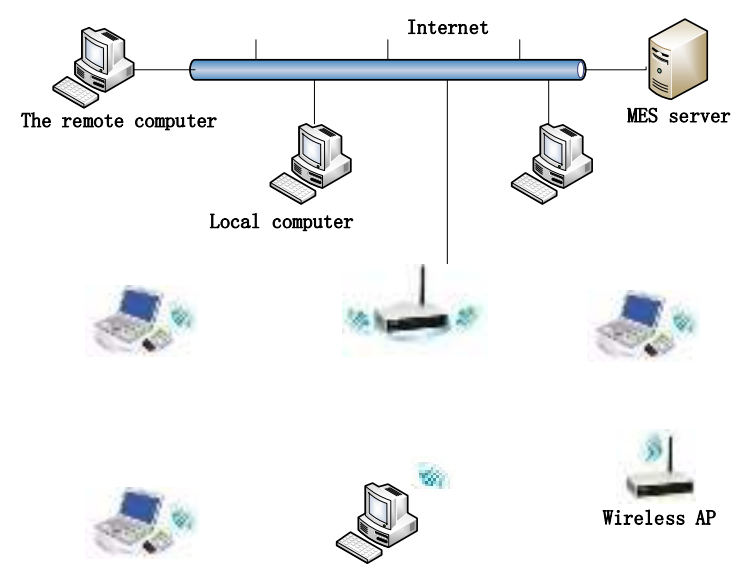

Figure 1. The wireless WIFI network

In the ZigBee protocol network, three device objects are defined: the coordinator, the router and the terminal equipment. The network mode is divided into three topology types: star structure, cluster tree structure and reticular formation (mesh) [5]. In an actual production process, the production process information monitoring nodes perform the routing function and terminal equipment. The mesh structure topology can expand the scope of the network in practical applications, and has a more flexible network structure than star structure and cluster tree structure topologies. The mesh structure provides the communication paths by self-organization and routing functions. This effectively reduces the information transmission delay and improves the reliability of the data communication net. The network transmission speed over the whole network may be up to $250 \mathrm{Kbps}$, and CAN bus communication can improve the efficiency of the data transmission by coordination of communication between nodes and the computer. CAN bus can achieve up $1 \mathrm{Mbps}$ transmission communication rate over short distances (less than 40m). Compared with [8], RS232 communication efficiency is higher. The network structure was as shown in Fig.2.

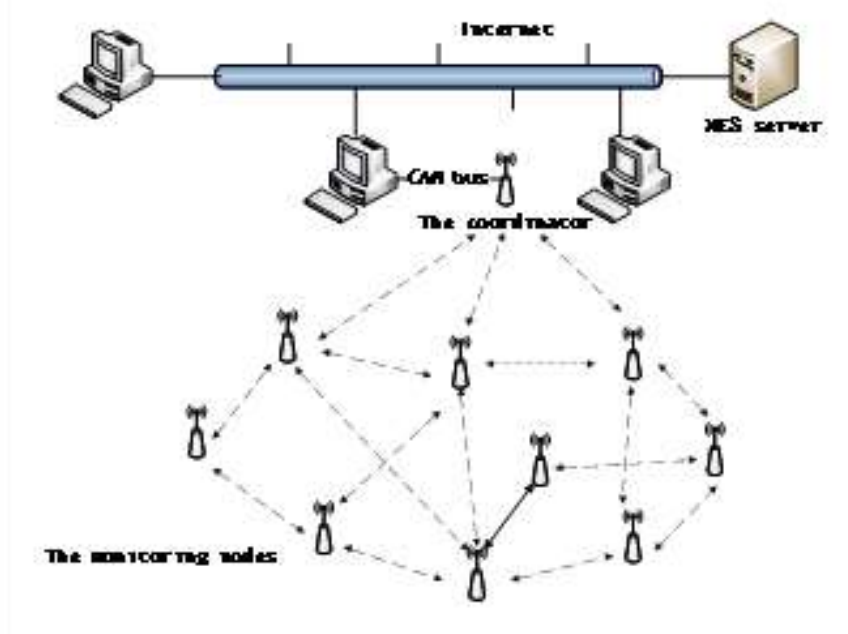

Figure 2. ZigBee network for information transmission

\section{B. Function of Monitoring network node}

Real-time information monitoring in the production process mainly includes information relating to people, equipment, materials, the production process and the manufacturing environment. The information on people relates to capturing information on the worker status, location and time. Equipment information mainly includes the real-time running status and the working condition of machine.Simultaneously, material information, the production process method, the working environment and other information, need to be monitored, as shown in Table 1 .

TABLE I. FUNCTION OF MONITORING NODES

\begin{tabular}{|c|c|c|c|c|}
\hline Monitoring & \multicolumn{4}{|c|}{ Monitoring content } \\
\hline People & $\begin{array}{l}\text { Personnel } \\
\text { position }\end{array}$ & Work content & $\begin{array}{l}\text { Working } \\
\text { time }\end{array}$ & $\begin{array}{l}\text { Work } \\
\text { efficiency }\end{array}$ \\
\hline Equipment & $\begin{array}{l}\text { Equipment } \\
\text { state }\end{array}$ & $\begin{array}{l}\text { Equipment } \\
\text { running time }\end{array}$ & $\begin{array}{l}\text { Equipment } \\
\text { power source } \\
\text { parameters }\end{array}$ & $\begin{array}{l}\text { Equipment } \\
\text { speed }\end{array}$ \\
\hline Material & $\begin{array}{l}\text { Material } \\
\text { number }\end{array}$ & $\begin{array}{l}\text { Material } \\
\text { quality }\end{array}$ & $\begin{array}{l}\text { Material } \\
\text { shortages }\end{array}$ & \\
\hline $\begin{array}{l}\text { Production } \\
\text { process }\end{array}$ & $\begin{array}{l}\text { Order } \\
\text { processing }\end{array}$ & & & \\
\hline $\begin{array}{l}\text { Manufacturing } \\
\text { environment }\end{array}$ & $\begin{array}{l}\text { Environment } \\
\text { temperature }\end{array}$ & $\begin{array}{l}\text { Environmental } \\
\text { humidity }\end{array}$ & $\begin{array}{l}\text { Dust } \\
\text { concentration }\end{array}$ & \\
\hline
\end{tabular}




\section{DESIGN OF ZIGBEE MONITORING NODES}

\section{A. ZigBee monitoring node hardware}

Table 1 shows the node functions required to realize each elemental function in production process monitoring. The monitoring node hardware functions are shown in Fig.3.

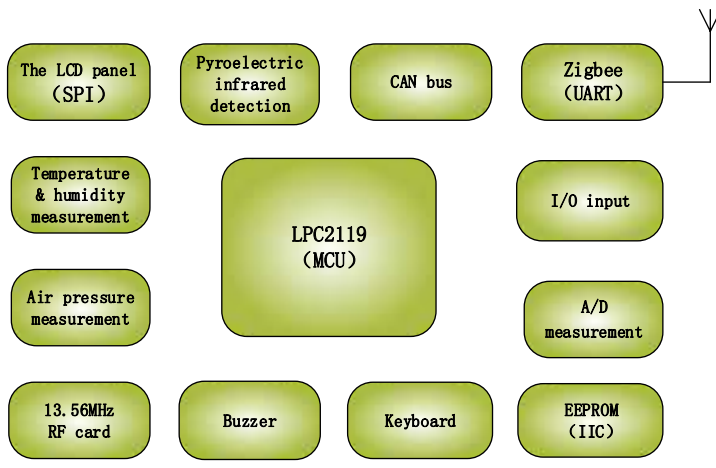

Figure 3. Functions of the monitoring node.

Analysis of the hardware functions shows that the hardware design includes the power supply, ZigBee protocol, RF card information transmission, $\mathrm{A} / \mathrm{D}$ data acquisition, and sensing circuit design.

Multiple CPUs are adopted for complex hardware function management in order to improve the working efficiency. For ZigBee communication transmission circuit design, the CC2530 system-on-chip from TI Company is a core component. This device realizes an integrated data transceiver wireless sensor network. The peripheral device is simple. The circuit mainly includes crystals and resistance capacitance inductance components such as a high frequency matching network. The antenna adopts external high-gain SMA rods, with a gain of $6 \mathrm{~dB}$, which ensures that the receiving sensitivity is relatively high. The CC2530 ZigBee network programming can configure the monitoring nodes automatically after an electric network failure. When part of the node fails, the network also has the ability to repair itself and each node can send and receive data. Communication data can be transmitted between any two nodes in the network.

The IC card circuit was designed for employees. A non-contact RF card is used and the IC card circuit is composed of an IC chip and sensing antenna, which are encapsulated into a PVC card. The IC circuit is a short distance of 5-10 $\mathrm{mm}$ from the surface of the card. Data is transmitted by radio waves. The circuit card reader antenna consists of lines of printed circuit boards, and the circuit is a single module. MF RC522 of NXP Company is a core chip in the circuit design for the card reader. Also, it is a single read card circuit module design. Data transmission uses a serial port for management of the CPU.

The A/D acquisition circuit design was designed at $0.5 \mathrm{~V}$ and $4.20 \mathrm{~mA}$ and shares the same circuit. It is based on the principle that the voltage is equal to the product of the current and the resistance $(\mathrm{V}=\mathrm{I} * \mathrm{R})$. The acquisition circuit terminal was designed with a diode and a capacitor for noise filtering, to improve the reliability of the circuit, as shown in Fig.4.

The LHi878 pyroelectric infrared sensor was chosen for the peripheral sensors for body detection. DHT22 is the temperature and humidity sensor of the monitoring node.

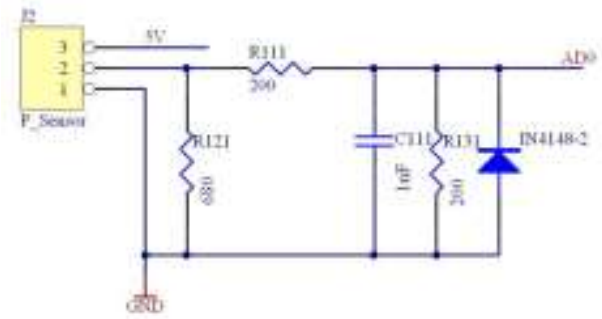

Figure 4. The A/D sampling circuit for voltage and current

The monitoring node installation location should be considered so that the node has a minimum effect on the operation of other equipment. Therefore, a multilayer circuit layout form was selected so that the whole structure was divided into two layers, maintaining a compact structure with a small size $(6 \mathrm{~cm} \times 6 \mathrm{~cm})$ which would be easy to install. The hardware circuit is shown in Fig .5.

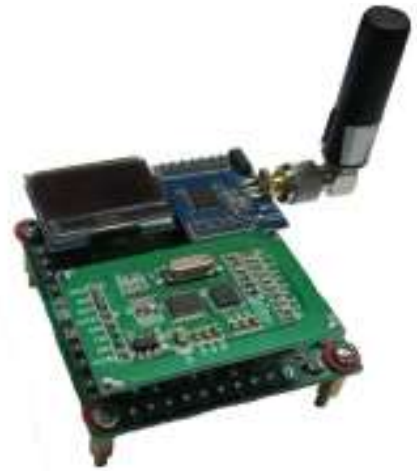

Figure 5. Double layer structure of the monitoring node $(6 \mathrm{~cm} \times 6 \mathrm{~cm})$

\section{B. Soft design of ZigBee monitoring nodes}

The software flow of data was as shown in Fig .6 for the ZigBee network structure. Each data acquisition node transmits data from its ZigBee coordinate nodes at the same time, in order to obtain real-time monitoring of the production process. The data uses CAN bus transfer to computer terminals. Finally, the data is transferred to the MES system server database.

The monitoring software flow from nodes is shown in Fig .7. Firstly, the system is powered on. The system parameters of the block are read into the EEPROM. Then the system initializes and the parameter information is displayed on in the 12864 LCD. The sensors obtain working parameters in the acquisition work environment at the same time, such as temperature and humidity, pressure equipment, etc. The data is transferred to the CPU by a preprocessing circuit. After workers swipe their ID card, ID information is sent to the server by the ZigBee block. The employee information is retrieved in the database and the worker status is sent back. The node receives the job number, and the job number is displayed on the LCD After the worker swipes the card number ID, it is sent to the server by the ZigBee block. The work information is retrieved in the database, which will be sent back to the worker. The received information about the working status 
is displayed on the LCD. The nodes use the work time monitoring equipment at the same time to record the worker's workload, and report the data to the server database. The node can receive instructions relating to the upper machine processing, in addition to entry through the keyboard to change part parameters, equipment status, or enter information such as product quality. Human-body pyroelectric infrared detection sensor monitors if there are any workers within the location. If nobody is near the node, the node will enter a dormant state and sleep.

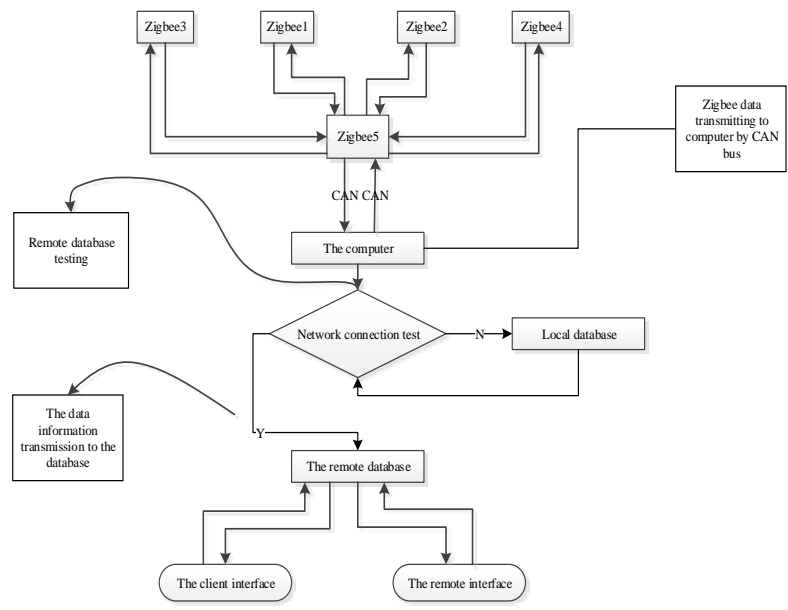

Figure 6. System flow chart of data

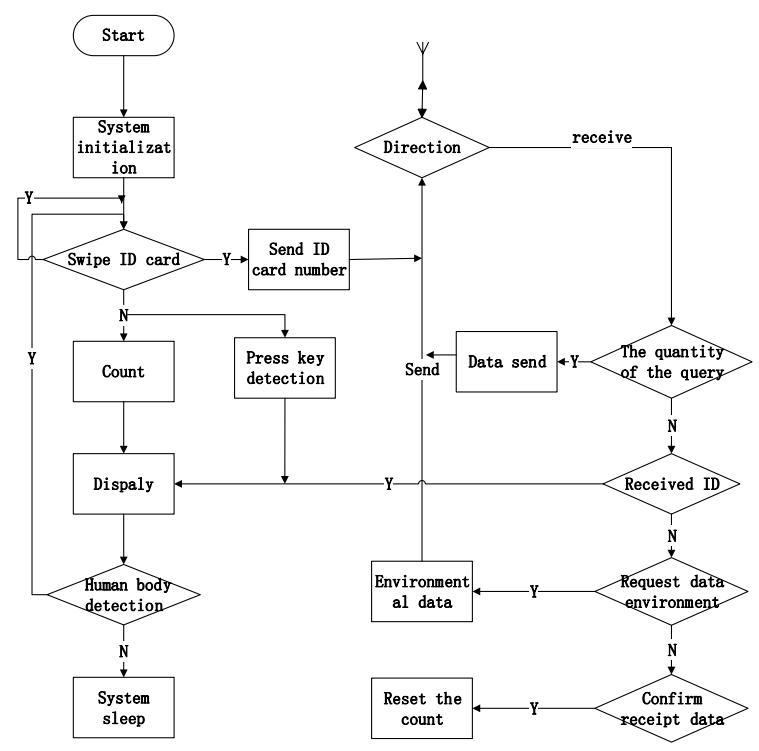

Figure 7. Software flow chart of the monitoring node

\section{AN EXPERIMENT OF NETWORK PERFORMANCE}

Using the above design, production of the actual product is tested using a ZigBee nodal network for data acquisition. The system performance test network has 15 nodes. For point-to-point data transmission, it was found that data transmission was achieved up to $110 \mathrm{~m}$. For data transmission from terminal nodes to coordinate nodes, the minimum time interval between data compartments was $18 \mathrm{~ms}$, and the packet loss rate was less than 5\%. As transmission distance increased, the transfer rate reduced and the packet loss rate increased. For data transmission from coordinate nodes to terminal nodes, the time interval between the two nodes was a minimum of $500 \mathrm{~ms}$. The packet loss rate was under $5 \%$, and the packet loss rate increased as the transmission distance increased.

The performance test confirms that the whole network data transmission can satisfy actual requirements. The disadvantage of using wireless communications is that it is easily affected by the influence of high voltage electromagnetic fields in some work environments. In realtime applications where this is a factor, data can be transferred using cable.

\section{CONCLUSIONS}

For discrete manufacturing process information monitoring, this paper proposed two types of wireless networks to collect data. The strategy was based on WIFI and ZigBee protocol wireless network with a combination of network modes. A ZigBee monitoring nodal network was designed, and used in a cable connector production testing network and node. The network data transmission speed and distance satisfy the cable requirements, even when capturing machine production information collection. Test results show that the test nodes can satisfy information collection for general production processes.

\section{ACKNOWLEDGMENT}

The authors thank the anonymous reviewers for their useful comments, and gratefully acknowledge the support of colleagues.

\section{REFERENCES}

[1] A.Ihsan, and H.V.Landeghem. "Evaluating the performance of discrete manufacturing process using RFID: A case study," Robotics and Computer-Integrated Manufacturing vol29.6, pp.502-512,2013.

[2] L.Na, T.Jie, and Z.Zhu. "Monitor and control system with RFID technology in discrete manufacturing line," RFID-Technology and Applications (RFID-TA), 2010 IEEE International Conference on. IEEE, 2010, pp.71-76.

[3] Z.Ray Y., et al. "RFID-enabled real-time manufacturing execution system for mass-customization production," Robotics and Computer-Integrated Manufacturing vol29.2, pp.283-292,2013.

[4] P.Figueiredo, et al. "Autonomy Suitability of Wireless Modules for Ambient Assisted Living Applications: WiFi, ZigBee, and Proprietary Devices," Sensor Technologies and Applications (SENSORCOMM), 2010 Fourth International Conference on. IEEE, pp.169-172, July 2010.

[5] IEEE Std 802.15.4 -2006 Wireless Medium Access Control (MAC) and Physical Layer (PHY) Specifications for Low-Rate Wireless Personal Area Networks (WPANs)[S]

[6] D.Costa, Cesar, and L. Reis. "RFID applications to the optimization of discrete manufacturing process," Signal Processing,Computing and Control (ISPCC), 2013 IEEE International Conference on. IEEE, 2013, pp.1-5.

[7] B.Castao and M.Rodriguez-Moreno. "A ZigBee and RFID hybrid system for people monitoring and helping inside large buildings," Industrial Electronics \& Applications (ISIEA), 2010 IEEE Symposium on. IEEE, 2010, pp.16-21.

[8] Y.Liuyin, et al. "The Research on Monitoring of Discrete Manufacturing Process Based on Internet of Things," Green Computing and Communications (GreenCom), 2013 IEEE and Internet of Things (iThings/CPSCom), IEEE International Conference on and IEEE Cyber, Physical and Social Computing. IEEE, 2013, pp.1186-1191. 
[9] Z.Ray Y., et al. "A two-level advanced production planning and scheduling model for RFID-enabled ubiquitous manufacturing," Advanced Engineering Informatics (2015).

[10] H.Fernández-López,et al. "Towards the design of efficient nonbeacon-enabled ZigBee networks," Computer Networks vol56.11, pp.2714-2725, 2012.
[11] T.Pan, and L.Xiaoyang. "Hybrid wireless communication system using zigBee and WiFi technology in the coalmine tunnels," Measuring Technology and Mechatronics Automation (ICMTMA), 2011 Third International Conference on. vol.2, pp.340-343, January 2011 